\title{
Discrimination analysis of human lung cancer cells associated with histological type and malignancy using Raman spectroscopy
}

\author{
Yusuke Oshima \\ Aoyama Gakuin University \\ College of Science and Engineering \\ Department of Chemistry and Biological Science \\ 5-10-1 Fuchinobe, Sagamihara \\ Kanagawa 229-8558, Japan \\ and \\ RIKEN \\ Optical Biopsy Development Research Unit \\ 2-1 Hirosawa, Wako \\ Saitama, 351-0198 Japan
}

\section{Hideyuki Shinzawa}

RIKEN

Optical Biopsy Development Research Unit

2-1 Hirosawa, Wako

Saitama, 351-0198 Japan

\section{Tatsuji Takenaka \\ Chie Furihata}

Aoyama Gakuin University

College of Science and Engineering

Department of Chemistry and Biological Science

5-10-1 Fuchinobe, Sagamihara

Kanagawa 229-8558, Japan

\section{Hidetoshi Sato}

Kwansei Gakuin University

School of Science and Technology

Department of Bioscience

2-1 Gakuen, Sanda

Hyogo, 669-1337 Japan

and

RIKEN

Optical Biopsy Development Research Unit

2-1 Hirosawa, Wako

Saitama, 351-0198 JapanE-mail: hidesato@kwansei.ac.jp

\begin{abstract}
The Raman spectroscopic technique enables the observation of intracellular molecules without fixation or labeling procedures in situ. Raman spectroscopy is a promising technology for diagnosing cancers-especially lung cancer, one of the most common cancers in humans-and other diseases. The purpose of this study was to find an effective marker for the identification of cancer cells and their malignancy using Raman spectroscopy. We demonstrate a classification of cultured human lung cancer cells using Raman spectroscopy, principal component analysis (PCA), and linear discrimination analysis (LDA). Raman spectra of single, normal lung cells, along with four cancer cells with different pathological types, were successfully obtained with an excitation laser at $532 \mathrm{~nm}$. The strong appearance of bands due to cytochrome $c(\mathrm{cyt}-\mathrm{c})$ indicates that spectra are resonant and enhanced via the Q-band near $550 \mathrm{~nm}$ with excitation light. The PCA loading plot suggests a large contribution of cyt- $C$ in discriminating normal cells from cancer cells. The PCA results reflect the nature of the original cancer, such as its histological type and malignancy. The five cells were successfully discriminated by the LDA. ๑) 2010 Society of Photo-Optical Instrumentation Engineers. [DOI: 10.1117/1.3316296]
\end{abstract}

Keywords: Raman spectroscopy; lung cancer; single cell; cancer; diagnosis; cytochrome $c$.

Paper 09431RR received Sep. 24, 2009; revised manuscript received Dec. 7, 2009; accepted for publication Dec. 9, 2009; published online Feb. 18, 2010.

\section{Introduction}

Lung cancer is the leading cause of cancer-related death worldwide. Early detection and appropriate treatment for cancerous lesions of patients can reduce the risk of death and improve the quality of life (QOL). Histopathological diagnosis of a lesion is necessary for physicians to decide on a therapeutic plan for a patient. Lung cancer is generally classified into two categories: small cell carcinoma (SC), and non-small cell carcinoma. This information reflects the characteristic of the tumor: SC is usually more aggressive with poorer differentiation potential than non-small cell carcinoma. Cancerous tissue of non-small lung cancer is further classified into three

Address all correspondence to: Hidetoshi Sato, Kwansei Gakuin University, School of Science and Technology, Department of Bioscience, 2-1 Gakuen, Hyogo 669-1337, Japan. Tel: 81-79-565-7228; Fax: 81-79-565-7228; E-mail hidesato@kwansei.ac.jp. major types according to histological tissue pattern: adenocarcinoma (AD), squamous cell carcinoma (SQ), and large cell carcinoma (LC). ${ }^{1-3}$ Information on tissue morphology and cancer cell type is important for confirming a diagnosis. Raman spectroscopy is a type of vibrational spectroscopy that provides useful information for estimating the molecular composition of a sample. If the molecular composition changes in cancer tissues, its Raman spectrum must reflect those changes.

Raman spectroscopy is an especially powerful, noninvasive analysis tool for tissue and cells for two major reasons. ${ }^{4-6}$ One advantage is that the bands that form due to water do not overlap with the fingerprint region. This is a large advantage over infrared and near-infrared (NIR) spectroscopy, because biological samples are usually rich in water content. The other advantage is that Raman spectroscopy uses light in the visible

1083-3668/2010/15(1)/017009/8/\$25.00 @ 2010 SPIE 
and NIR regions, which permits the use of highly sensitive and useful optical devices in the development of Raman instruments, such as optical fiber and charge-coupled device (CCD) detectors. Haka et al. demonstrated Raman spectroscopy in the diagnosis of breast cancer in vivo. ${ }^{7}$ They differentiated breast tissue into four pathological typesfibroadenoma, infiltrating carcinoma, fibrocystic change (not malignant), and normal-by using a model fitting the basic Raman spectra normalized to the sum total contribution of one. They also demonstrated Raman spectroscopy for intraoperative margin assessment of breast cancer in vivo. ${ }^{8}$ Huang et al. measured bronchial tissue specimens, normal cells, squamous cell carcinoma, and adenocarcinoma. They found that differences between normal and tumor tissues seemed to arise from protein and lipid conformational changes and concentration of nucleic acid. ${ }^{9}$ Min et al. demonstrated that 1064-nm-excitation NIR Raman spectroscopy could be employed to collect Raman spectra from fresh human lung tissue, which was found to emit strong fluorescence-interfering Raman signals under visible excitation. ${ }^{10}$ Yamazaki et al. observed the amide I band on the Raman spectra, which is characteristic of cancerous tissue. They found that the amide I band was assignable to collagen-like material on the basis of their previous research, in which they used formalin-fixed tissue. ${ }^{11}$ Magee et al. evaluated Raman spectroscopy in the diagnosis of lung cancer using a Raman mini-probe during bronchoscopy ex vivo. ${ }^{12}$ These reports have revealed that $\mathrm{Ra}-$ man spectroscopy has a high enough potential for the practical diagnosis of various types of cancer.

The identification of leukemia cells with Raman spectroscopy has been reported by Chan et al. ${ }^{13,14}$ They measured the Raman spectra of T- and B-lymphocyte cells, and classified them into normal and cancerous groups by principal component analysis (PCA). The results indicated that differences in DNA and protein were responsible for discrimination via PCA. Changes in gene expression and enzyme activity in metabolic cycles are important signals for understanding the mechanism of cancer occurrence. ${ }^{1-3,15,16}$ Swain et al. demonstrated cell cycle analysis with microscopic Raman spectroscopy. ${ }^{17}$ Notingher et al. reported that, in Raman spectral dynamics of differentiation in embryonic stem (ES) cells, Raman bands of RNA contribute to distinguishing between differentiated and undifferentiated states. ${ }^{18}$

In the present study, Raman spectroscopy was applied to the analysis of differences among cultured cells of one normal cell and four lung cancer cell lines. Raman measurement was carried out directly on a culture dish with minimal treatment. Multivariable analyses were performed to discriminate among cells and to investigate the origin of differences. An excitation wavelength of $532 \mathrm{~nm}$ was employed instead of 785 or $633 \mathrm{~nm}$. Although it has been reported that visible excitation has the disadvantages of photodegradation and fluorescence, ${ }^{19,20}$ the $532-\mathrm{nm}$ line provides resonance enhancement of cytochrome $c$ (cyt-c), which cannot be observed with excitation wavelengths of 785 and $633 \mathrm{~nm}$. Our final purpose was the diagnosis of cancer using Raman spectroscopy. Spectra for tissue included information about the cancer cells and the extracellular matrix. We assumed that the composition and property of the extracellular matrix depended on the individual history of the disease, which can
Table 1 List of human lung cell lines used in Raman measurement.

\begin{tabular}{llll}
\hline $\begin{array}{l}\text { Name of } \\
\text { cell line }\end{array}$ & $\begin{array}{l}\text { Cancer/ } \\
\text { Normal }\end{array}$ & Histological type & Malignancy \\
\hline MRC-5 & Normal & - & - \\
RERF-LC-MS & Cancer & Adenocarcinoma & Low-Med \\
EBC-1 & Cancer & Squamous cell carcinoma & Low-Med \\
Lu-65 & Cancer & Large cell carcinoma & High \\
RERF-LC-MA & Cancer & Small cell carcinoma & High \\
\hline
\end{tabular}

make a clear diagnosis difficult with Raman spectroscopy. Diagnosis can be easier if it is possible to obtain information from the cells alone. Hence, the present study focuses on cancer cells and demonstrates the performance of Raman spectroscopy for the diagnosis and discrimination of the single cell.

\section{Materials and Methods}

\subsection{Cell Cultures}

All cell lines were purchased from the Japanese Collection of Research Bioresources (JCRB) cell bank. Cell lines included one normal lung cell line [MRC-5 (JCRB9008)] and four cancerous strains [RERF-LC-MS (JCRB0081), EBC-1 (JCRB0820), Lu-65 (JCRB0079), and RERF-LC-MA (JCRB0812)]. Each cancerous cell strain was of a different histological type (Table 1): AD for RERF-LC-MS, SQ for EBC-1, LC for Lu-65, and SC for RERF-LC-MA. The cell lines MRC-5, RERS-LC-MS, EBC-1, and RERS-LC-MA were seeded into Eagle's minimum essential medium (51200038; Invitrogen Corp., Carlsbad, California, USA) with $1.0 \%$ (v/v) nonessential amino acids (11140-050), $292 \mathrm{mg} / \mathrm{L}$ L-glutamine (21051-024), and 10\% (v/v) heat-inactivated fetal bovine serum (10082-147). Lu-65 was seeded into the RPMI1640 medium (11835-030) with nonessential amino acids and fetal bovine serum in the same manner. The cells were maintained at $37{ }^{\circ} \mathrm{C}$ with $5 \% \mathrm{CO}_{2}$ within six days on a synapse fine-view dish (SF-S-D27; Fine Plus International Ltd., Japan), the bottom of which was made of silica glass for spectroscopic measurement. For cell viability tests, a Trypan blue dye method [0.4\% (w/v), 15250-061; Invitrogen] was used a few minutes after the Raman measurement.

\subsection{Raman Measurement}

Raman spectra were obtained using a laser Raman microscope (in Via Raman microscope; Renishaw plc, UK). An excitation laser (Nd: YAG SHG) of $532 \mathrm{~nm}$ with power of $50 \mathrm{~mW}$ was focused on a single cell via a $50 \times$ objective lens with numerical aperture $(\mathrm{NA})=0.75$. Raman scattering light from the sample was collected using the same objective lens and detected by a Peltier-cooled CCD $(0.5$ inch, $576 \times 384$ pixels $)$ with a grating $(1800$ lines $/ \mathrm{mm})$ for the visible region and a 60 -s exposure time. The spectral resolution was less than $2 \mathrm{~cm}^{-1}$, and the diameter of the laser spot at the focus point was less than $1 \mu \mathrm{m}$. The culture medium was removed, and 
the cells were washed twice with a buffer solution (Hanks' balanced salt solution, 14175-095; Invitrogen, Carlsbad, California, USA) before the Raman measurement. Five Raman spectra were randomly taken in each dish. Background spectra were obtained by focusing on a vacant region on each dish where no cell existed. Raman spectra measurements with excitation wavelengths of $633 \mathrm{~nm}$ and $785 \mathrm{~nm}$ were obtained using the Nanofinder ${ }^{\circledR}$ (Tokyo Instruments, Inc., Japan) equipped with a 600 lines/mm holographic grating blazed at $750 \mathrm{~nm}$. For analysis of the concentration of cyt- $c$, Raman measurements of 20 cells each of MRC- 5 and RERF-LC-MS were carried out with a $60 \times$ water-immersion objective lens $(\mathrm{NA}=1.20)$, and exposure time was $30 \mathrm{~s}$. Purified cyt- $c$ from bovine heart (c2037; Sigma, Japan) was solved into distilled water as an oxidized cyt- $c$ solution. A reduced cyt- $c$ solution was prepared by adding bisulfate mix (QIAGEN, Japan) into the cyt-c solution.

\subsection{Data Analysis}

Raman spectral data analysis was performed with the commercially available GRAMS software (Galactic Industries Corp., Salem, New Hampshire, USA). The background spectrum was subtracted from the raw Raman spectrum, and the intensity of individual Raman spectra was normalized with a band of $1003 \mathrm{~cm}^{-1}$ of phenylalanine. To verify the concentration of cyt- $c$, we used the Raman spectra of 20 cells each of MRC-5 and RERF-LC-MS. The averaged intensity of the band at $1128 \mathrm{~cm}^{-1}$ relative to the band at $1003 \mathrm{~cm}^{-1}$ and its standard error in each cell strain was calculated, and its significance in difference was confirmed with Student's $t$-test. For chemometrics analysis, a Raman spectral dataset was prepared for PCA and linear discrimination analysis (LDA). Sirius 7.1 software (Pattern Recognition Systems, Norway) was employed for PCA. Before analysis, the data were treated with mean-centering, and a classification model was constructed with two principal components (PCs). The third component in all models had minimal or no important information when judged by the loading weight of PC3. Two-dimensional plots showed scores for PC1 ( $x$ axis) and PC2 ( $y$ axis). LDA was calculated by the SPSS Statistics 17.0 software (SPSS Japan Inc., Japan). We used a predictive model composed of the discrimination function based on the linear combination of predictor variables that provide the best discrimination among cell strains. Independent variables used to build the discrimination model were selected according to the PCA loading weight. The LDA model was also built with the independent variables selected by the step-wise method (which obtains independent variables automatically) for optimal results. All predictive models were cross-validated by the leave-one-out method to evaluate its accuracy.

\section{Results and Discussion}

\subsection{Raman Spectroscopy}

Microscopic Raman spectroscopy has been performed for biochemical characterization of single living cells. In our study, with Raman spectroscopy, we succeeded in obtaining high signal-to-noise Raman spectra of cells without fixation. There was no interference of fluorescence from the dish, the buffer, or the cell itself. An excitation laser was focused within an

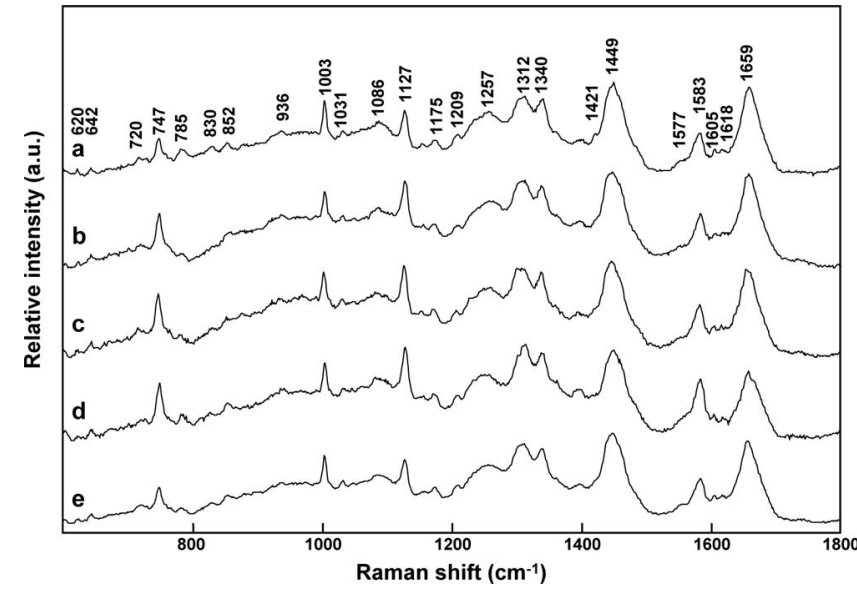

Fig. 1 Average Raman spectra of five cells: (a) MRC-5 (normal cell line), (b) RERF-LC-MS (AD cell line), (c) EBC-1 (SQ cell line), (d) Lu-65 (LC cell line), and (e) RERF-LC-MA (SC cell line).

adherent cell. Raman signals from the substrate of the culture dish (made of quartz) appeared on the raw Raman spectra (data not shown). The quartz had broad Raman bands near 795,1060 , and $1200 \mathrm{~cm}^{-1}$. The background spectra, obtained from the vacant space of each cell culture dish, had Raman bands arising from the quartz window and a band at $1640 \mathrm{~cm}^{-1}$ due to water. Accordingly, the background spectrum was subtracted from the sample spectra to remove unwanted Raman bands. ${ }^{21}$ Figure 1 shows the Raman spectra of the following cell lines: (a) MRC-5 (normal cell line), (b) RERF-LC-MS (AD cell line), (c) EBC-1 (SQ cell line), (d) Lu-65 (LC cell line), and (e) RERF-LC-MA (SC cell line). The spectra in Fig. 1 included the mean spectra of five measurements in each culture dish to eliminate the effects of site dependency and cell cycles. The strong band at $1659 \mathrm{~cm}^{-1}$ seemed to consist of the amide I band of protein and the $\mathrm{C}=\mathrm{C}$ stretching band of lipids. The features at 1449, 1257, 1003 , and $936 \mathrm{~cm}^{-1}$ were assigned to the $\mathrm{CH}_{2}$ deformation, the amide III, the symmetric ring breathing bands of phenylalanine of the protein, and C-C stretching, respectively. ${ }^{6,13,17-24}$ Aromatic amino acid residues, phenylalanine, tyrosine, and tryptophan were expected to have weak and sharp Raman bands at 620, 642, 852, 1175, 1209, 1605, and $1618 \mathrm{~cm}^{-1}$. The bands at $720,785,830,1086,1340$, 1421 , and $1577 \mathrm{~cm}^{-1}$ were assigned to nucleic acids (DNA and RNA). ${ }^{6,13,17-24}$ Strong bands at 747,1127 , and $1583 \mathrm{~cm}^{-1}$ were assigned to cyt- $c .^{25,26}$ Figure 2 compares the spectra of pure cyt-c, as reduced [Fig. 2(d)], and oxidized [Fig. 2(e)]. Bands observed in the cell spectra indicate that cyt- $c$ in cells mostly exist in a reduced state. Raman spectra of EBC-1 were measured with excitations of $785 \mathrm{~nm}$ [Fig. 2(a)], and $633 \mathrm{~nm}$ [Fig. 2(b)]. Cyt- $c$ bands were not observed in these spectra, strongly suggesting that bands due to cyt- $c$ are resonant enhanced via Q-bands from 510 to $550 \mathrm{~nm}$, generally observed in the absorption spectrum of cyt- $c$, with a 532-nm excitation wavelength (Fig. 2).

All the cell strains had the adherence property; this cell adherence indicated healthy growth on the culture dish. ${ }^{21} \mathrm{Ra}-$ man measurement was carried out to the confluent cell sheet. After the Raman measurements, cell morphology and cell ad- 


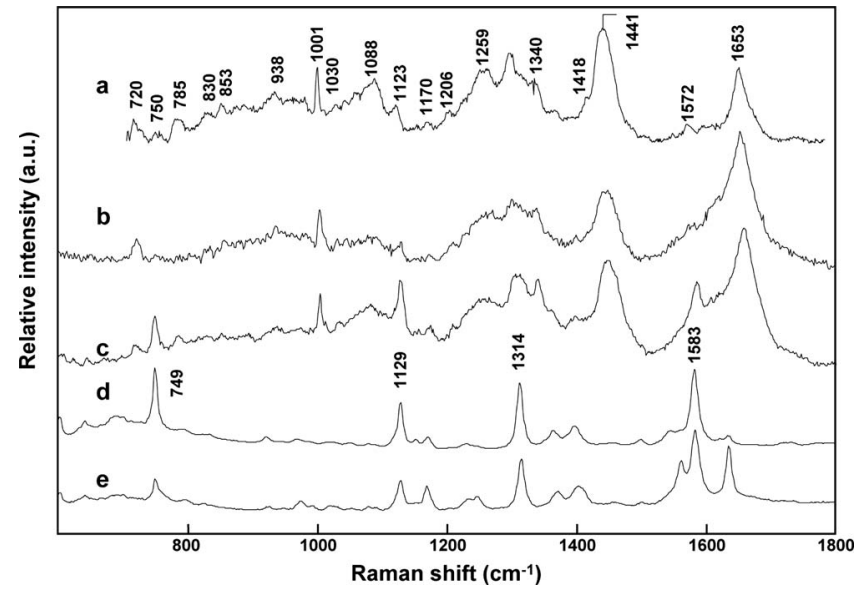

Fig. 2 Raman spectra of EBC-1 measured with excitation wavelengths of (a) 785, (b) 633, and (c) $532 \mathrm{~nm}$. Spectra of (d) reduced, and (e) oxidized states of cyt-c.

herence did not change. The results of the Trypan blue dye staining examination were also negative..$^{20,22,27}$ Hence, we concluded that the cells did not die by using Raman measurement with 532-nm laser irradiation $(50 \mathrm{~mW}, 60 \mathrm{~s})$. Notingher et al. reported that 488-nm and 514-nm irradiation induced damage to the cell at $5 \mathrm{~mW}$ of laser power and 5 to $20 \mathrm{~min}$ of exposure. ${ }^{20}$ Spectra of a particular cell were measured repeatedly to estimate the damage threshold for the 532-nm laser exposure at $50 \mathrm{~mW}$ of laser power. This measurement was carried out for four cells. The bands due to cyt- $c$ showed no change up to $210 \mathrm{~s}$ (data not shown) in every cell. A band at $1003 \mathrm{~cm}^{-1}$, assigned to phenylalanine, showed a reduction in the band intensity for measurements after $210 \mathrm{~s}$ of exposure time in two cells, but the other two cells did not show spectral changes even after $210 \mathrm{~s}$. No spectral changes were observed in the other bands up to $210 \mathrm{~s}$ of exposure time.

On initial observation, the Raman spectral features of the cells were similar, which suggests that there was no significant difference in the overall molecular composition of the lipids and proteins in the normal and cancer cells. The intensity ratio of the amide I band at $1660 \mathrm{~cm}^{-1}$ to the $\mathrm{CH}$ bending band at $1440 \mathrm{~cm}^{-1}$ (amide I/CH bend) was high in the spectra measured with excitation wavelengths of 532 and $633 \mathrm{~nm}$. This may be due to the water band at $1640 \mathrm{~cm}^{-1}$ overlapping with the amide I band. The measuring site of the culture dish was altered on every measurement. The laser spot size (which depended on the objective lens) was estimated to be $\sim 1 \mu \mathrm{m}$ in diameter at the focus point, which was rather small compared to the $\sim 10-\mu \mathrm{m}$ cell size. It was assumed that each spectrum before averaging reflected the information of localized cytoplasmic components.

To analyze the small differences between normal and cancer cells in mean spectra, we calculated the subtracted spectra of normal and cancer cells. Figure 3 depicts differences between the spectra of four strains in relation to the MRC-5 (normal) strain: (a) RERF-LC-MS, (b) EBC-1, (c) Lu-65, and (d) RERF-LC-MA. The sharp band of phenylalanine at $1003 \mathrm{~cm}^{-1}$ was used as the standard for subtraction. Strong bands at 748,1129 , and $1586 \mathrm{~cm}^{-1}$ were assigned to cyt- $c$, as shown in Figs. 3(a)-3(c). Cyt-c, when associated with elec-

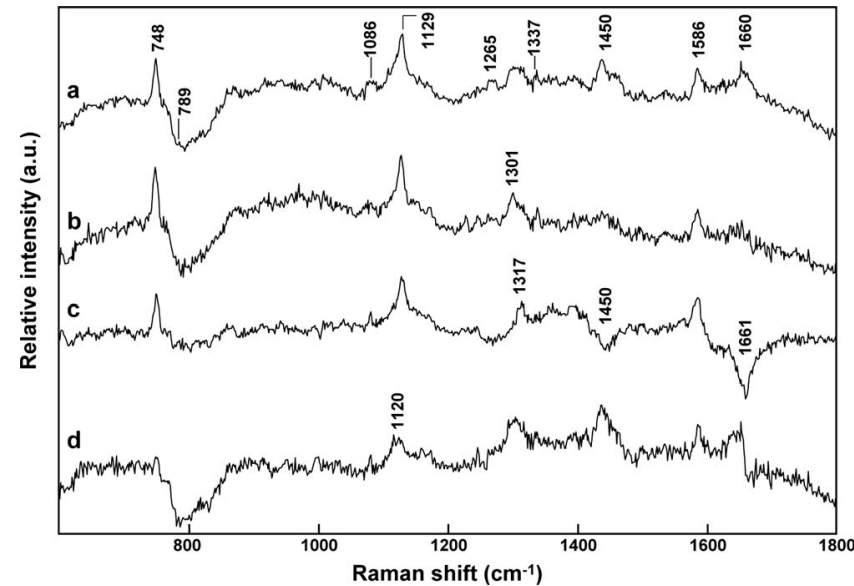

Fig. 3 Difference spectra between the normal cell (MRC-5) and the following cancer cells: (a) RERF-LC-MS, (b) EBC-1, (c) Lu-65, and (d) RERF-LC-MA.

tron transfer in oxidative phosphorylation, is usually localized at the mitochondrial inner membrane in the cytoplasm. The intensity of these Raman signals seems to be associated with the amount of the mitochondrial contents and the distribution of mitochondria in the cell as reported by Hamada et al. ${ }^{26}$ The strong appearance of these bands suggests that the cancer cells were rich in cyt- $c$ relative to phenylalanine, and possibly rich in mitochondrial cyt- $c$ or in mitochondria compared to MRC-5 (normal cells). Figure 4 compares the concentration of cyt- $c$ in MRC-5 and RERF-LC-MS calculated from the band intensity of their Raman spectra. Data are presented as the average and standard deviation (SD) of the mean. Significance was determined using Student's $t$-test on the basis of the 40 cells ( 20 cells each of MRC- 5 and RERF-LC-MS), and

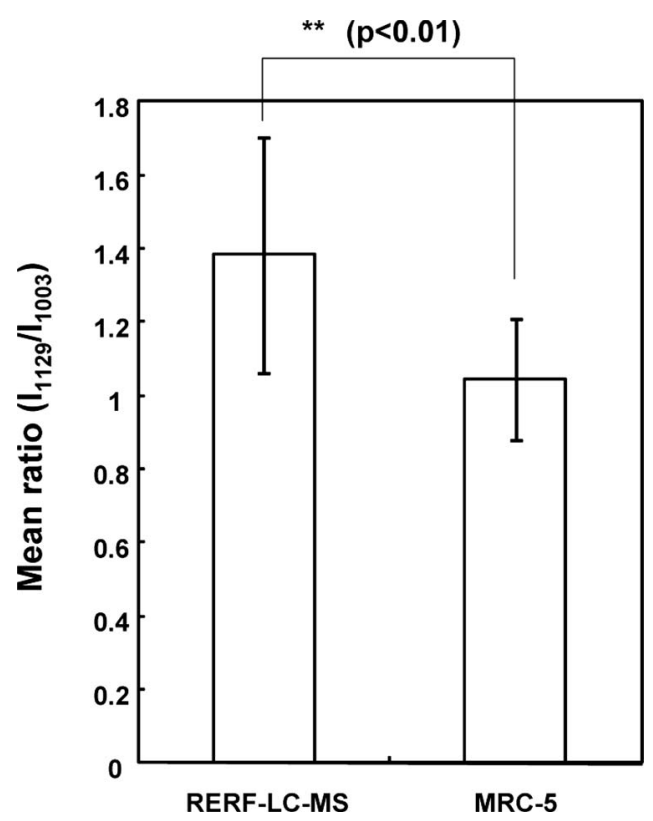

Fig. 4 Mean ratio of the $\mathrm{cyt}-\mathrm{C}$ band intensity at $1129 \mathrm{~cm}^{-1}$ to $1003 \mathrm{~cm}^{-1}\left(\mathrm{I}_{1129} / \mathrm{I}_{1003}\right)$ in RERF-LC-MS and MRC-5. Data are presented as the average \pm the SD of the mean. 
the $p$-value was 0.00012 . Values of $p<0.01$ were considered to be statistically significant. The Raman signal intensity of cyt- $c$ of RERF-LC-MA was weak, as shown in Fig. 3(d), suggesting that there was no significant difference in the concentration of cyt- $c$ between the normal cell and RERF-LC-MA.

A broad negative band near $800 \mathrm{~cm}^{-1}$, which appears in Figs. 3(a), 3(b), and 3(d), seems to be a baseline fluctuation due to the quartz substrate of the culture dish. Positive bands at 1660,1450 , and $1260 \mathrm{~cm}^{-1}$ were observed in the difference spectra of RERF-LC-MS [Fig. 3(a)] and RERF-LC-MA [Fig. 3(d)]. Negative bands at 1660 and $1450 \mathrm{~cm}^{-1}$ were observed in the spectrum of Lu-65 [Fig. 3(c)]. Judging from the band features and wave numbers, these bands were apparently assigned to proteins and/or lipids, and their composition in these cells differed from a normal composition. However, it was difficult to identify the types of proteins and lipids as increasing and decreasing in the cancer cells. Weak positive bands due to nucleic acids were barely observed at 789, 1086, 1265, and $1337 \mathrm{~cm}^{-1}$ in the subtraction spectra, which suggests that the chromatin ratio (nucleic acid/protein) was higher in the cancer cells than in the normal cell. However, the intensities of these bands were too weak to analyze the chromatin ratio quantitatively.

\subsection{Principal Component Analysis}

PCA classification models with a dataset for the normal cell and each cancer cell. The two spectral regions from $760 \mathrm{~cm}^{-1}$ to $870 \mathrm{~cm}^{-1}$, and from $1000 \mathrm{~cm}^{-1}$ to $1110 \mathrm{~cm}^{-1}$, seem to have been affected by the Raman band of quartz; therefore, the corresponding variables were excluded from the dataset. Figure 5 shows the score plots of PC1 ( $x$ axis) and PC2 ( $y$ axis) calculated for MRC-5 versus (a) RERF-LC-MS, (b) EBC-1, (c) Lu-65, and (d) RERF-LC-MA. In the score plots, the cancer cells were obviously distinguishable from the normal cell.

PC1 was the main contributor to the discrimination of cancer cells (RERF-LC-MS, EBC-1, and Lu-65) from the normal cell (MRC-5) in the score plots of Figs. 5(a)-5(c). For discrimination of the SC cell (RERF-LC-MA), both PC1 and PC2 were necessary. The contribution ratio of PC1 was typically more than $55 \%$ in all models. The loading plots of PC1 in these PCA models are depicted in Figs. 6(a)-6(d). The features in the plot curves closely resemble the difference spectra in Fig. 3. Although PC2 was not necessary to discriminate cancer cells (RERF-LC-MS, EBC-1, and Lu-65) from the normal cell, the typical contribution ratio was near $20 \%$, suggesting that the information extracted from PC2 reflected site dependency in the cytoplasm and in the cell cycle of each cell. In the PC1 loading plots of Fig. 6, the bands were assigned to cyt $-c$ at 748,1129 , and $1586 \mathrm{~cm}^{-1}$ [Figs. 6(a)-6(c)]. This indicates that the cancer cells were rich in cyt-c, so these bands contributed to the discrimination of cancer cells (RERF-LC-MS, EBC-1, and Lu-65) from the normal cell (MRC-5).

PC2 was necessary in addition to PC1 for discriminating RERF-LC-MA cancer cells from normal cells in spite of the low contribution rate of $16.4 \%$. This feature of the PC1 loading plot curve is similar to the difference spectrum of Fig. $3(\mathrm{~d})$. The bands at 1317,1450 , and $1661 \mathrm{~cm}^{-1}$ appear to have been assigned to proteins. Although lipids have bands close to

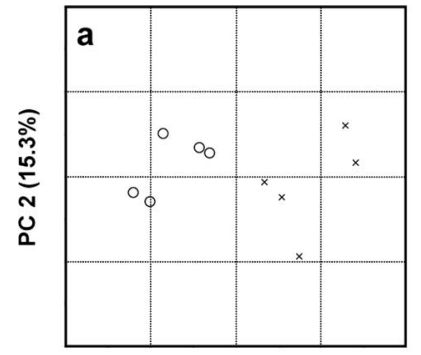

PC1 $(65.2 \%)$

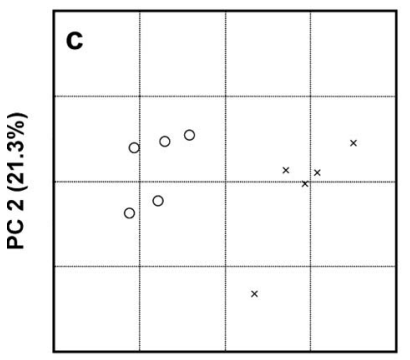

PC $1(64.1 \%)$

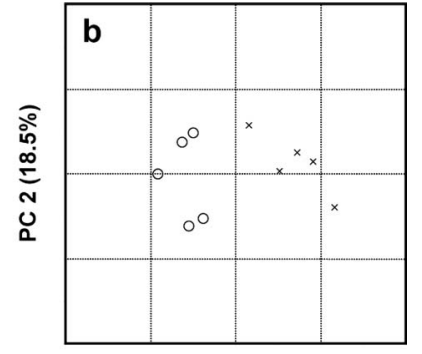

PC $1(57.4 \%)$

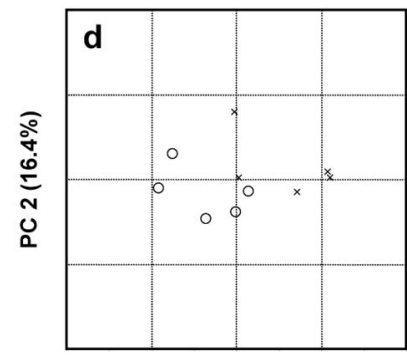

PC $1(62.0 \%)$
Fig. 5 Score plots of PCA models built with PC1 ( $x$ axis) and PC2 ( $y$ axis). PCA models were employed for the following Raman spectral datasets: (a) MRC-5 and RERF-LC-MS, (b) MRC-5 and EBC-1, (c) MRC-5 and Lu-65, and (d) MRC-5 and RERF-LC-MA. The contribution ratio of the total information for each component is shown in each component's axis label. $\bigcirc$ indicates normal cells; $\times$ indicates cancer cells.

these wave numbers, we estimated that the contribution of lipids was considerably weak because the $\mathrm{C}=\mathrm{O}$ stretching band of ester was absent in the curve. bands due to cyt- $c$ appear in the PC2 loading plot instead of the PC1 loading plot, as shown in Fig. 6(e). These observations suggest that the cancer cell RERF-LC-MA induced a conformational change and/or the production of a special protein.

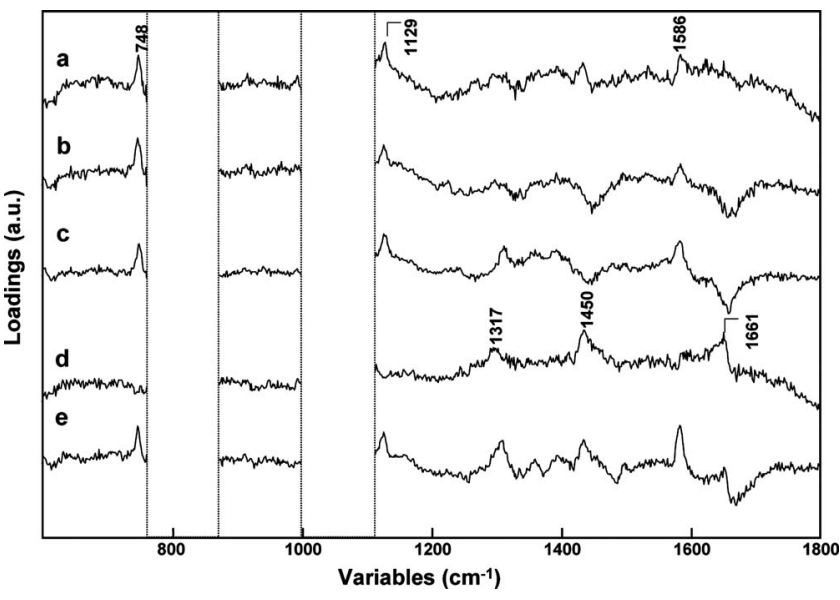

Fig. 6 Loading weight of PC1 in the following PCA models: (a) MRC-5 and RERF-LC-MS, (b) MRC-5 and EBC-1, (c) MRC-5 and Lu-65, and (d) MRC-5 and RERF-LC-MA. Datasets were excluded from PCA analysis in the spectral ranges of $760-870 \mathrm{~cm}^{-1}$ and $1000-1110 \mathrm{~cm}^{-1}$ because the spectra had a strong contribution of window material in the culture dish. 


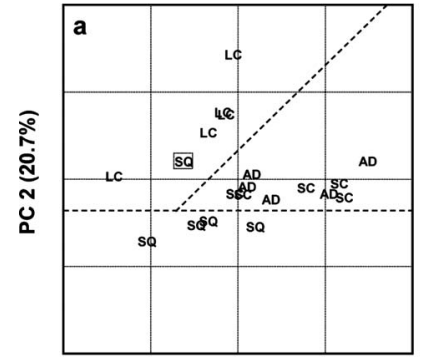

PC 1 (44.4\%)

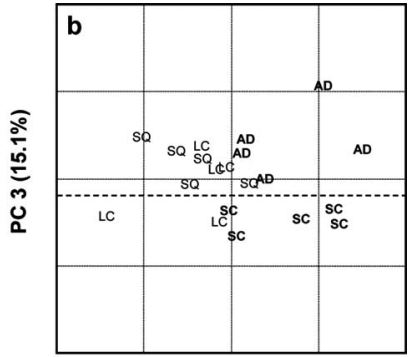

PC $1(44.4 \%)$
Fig. 7 Score plots built with (a) PC1 ( $x$ axis) and PC2 ( $y$ axis), and (b) PC1 $(x$ axis) and PC3 ( $y$ axis) for PCA models calculated from cancer cells RERF-LC-MS (AD), EBC-1 (SQ), RERF-LC-MA (SC), and Lu-65 (LC). The contribution ratios of PC1, PC2, and PC3 are shown in each axis label.

Using a discrimination plot, it is possible to estimate the malignancy of these cancers in the Raman spectrum. Figure 7 (a) depicts score plots of the PCA classification model of the four cancer cells: RERF-LC-MS (AD), EBC-1 (SQ), Lu-65 (LC), and RERF-LC-MA (SC). The four cancer cell types were almost successfully discriminated in the PCA model. In the score plot of PC1 and PC2, cells were classified into three groups - the SQ group, the LC group, and the group of AD plus SC-except for one SQ cell, which was classified in the LC group. In the score plot of PC1 and PC3, the AD and SC cells were well separated along with the PC3 axis [Fig. 7(b)]. $\mathrm{SC}$ is a type of poorly differentiated cancer, and it is generally classified as a high-grade malignancy. LC is also known as a poorly differentiated cancer with high-grade malignancy. AD and SQ cancers are well-differentiated cancers, and their malignancy is relatively low. ${ }^{2}$ loading plots of PCs are depicted in Fig. 8. PC1 [Fig. 8(a)] shows bands at $1660 \mathrm{~cm}^{-1}$ and $1440 \mathrm{~cm}^{-1}$, which were assigned to lipids and/or proteins; these differences could be related to cell morphology or to differences in protein and/or lipid production in these cells. PC2 [Fig. 8(b)] shows strong bands originating from cyt-c

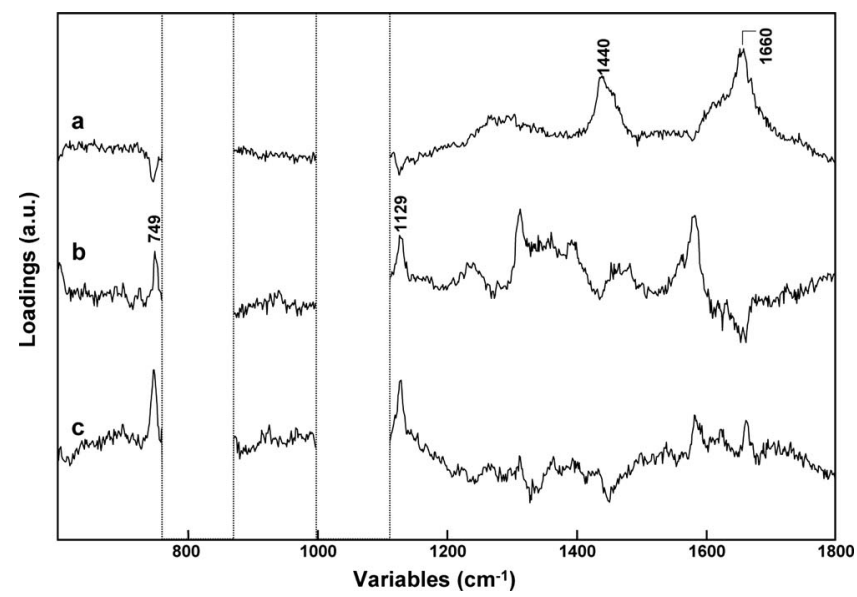

Fig. 8 Loading plots of (a) PC1, (b) PC2, and (c) PC3 for the PCA classification model in Fig. 7. Datasets were excluded from PCA analysis in the spectral ranges of $760-870 \mathrm{~cm}^{-1}$ and $1000-1110 \mathrm{~cm}^{-1}$ because the spectra had a strong contribution of window material in the culture dish.
Table 2 Results of LDA for cancer cells and normal cells.

\begin{tabular}{lll}
\hline & & Group of predictive model \\
\cline { 2 - 3 } Group of histological type & Normal & Cancer \\
\hline Normal & 5 & 0 \\
Adenocarcinoma & 0 & 5 \\
Squamous cell carcinoma & $1^{a}$ & 4 \\
Large cell carcinoma & 0 & 5 \\
Small cell carcinoma & $4^{a}$ & 1 \\
\hline Accuracy (normal or cancer) & & $80 \%$ \\
\hline aFalse-negative on the discrimination model
\end{tabular}

and several small bands assignable to nucleic acids. PC3 shows strong bands at $1129 \mathrm{~cm}^{-1}$ and $749 \mathrm{~cm}^{-1}$. Judging from their wave numbers, these bonds could be assigned to cyt- $c$, but PC3 did not produce bands at $1583 \mathrm{~cm}^{-1}$ and $1314 \mathrm{~cm}^{-1}$. We could not determine the reason that this spectral change took place in the cancer cells. It may reflect a change in the ratio of cyt- $c$ to other cytochromes or a change in the protein-cyt- $c$ interaction in the cell.

\subsection{Linear Discrimination Analysis}

LDA was used to discriminate cancer cells from normal cells. To construct the discrimination model, the intensities of cyt- $c$ bands at 747,1127 , and $1583 \mathrm{~cm}^{-1}$ (Table 2) were selected as the independent variables. Five normal cells and 20 cancerous cells, including $\mathrm{AD}, \mathrm{SQ}, \mathrm{LC}$, and $\mathrm{SC}$, were tested on the prediction model on the basis of the discrimination function. One SQ cell and four SC cells were classified into the normal group (false negative). The accuracy of the discrimination analysis was $80 \%$. The results suggest that Raman bands of cyt- $c$ are a potentially useful marker to distinguish normal lung cells from lung cancer cells except for SC cells. These results are consistent with those for PCA. For optimal classification, we employed the variable selection method of stepwise LDA. Thirteen variable datasets were automatically selected by the step-wise seeking method to maximize the discrimination efficiency of the cell groups. Five cell groups (normal, AD, SQ, LC, and SC) were successfully separated into five groups. The discrimination accuracy, determined by cross-validation, was $100 \%$, which suggests that LDA used with the step-wise selection method is capable of extracting an effective classifier for cell histology. The 13 variables included comprehensive information on cyt- $c$, proteins, nucleic acids, and lipids. In this analysis, the SC group laid in the region closest to the normal cell group on the discrimination map (due to the fact that SC cells have a spectrum similar to normal cells).

The pathological diagnosis to characterize the histological type of tumor tissue is generally based on morphological information and the nucleus and cytoplasm (N/C) ratio of biopsied tissues, as well as the size and depth of the cancer. ${ }^{2,7,8}$ However, the present study strongly suggests that the lipids and/or proteins composition and the concentration of cyt- $c$ 
can be reliable markers to discriminate the histological type and malignancy of the cancer cells. These markers are detectable in live single cells using Raman spectroscopy with the 532-nm excitation wavelength.

\section{Conclusions}

In this study, microscopic Raman spectroscopy was applied for direct measurement of human lung cancer cells in a culture dish in situ. High-quality Raman spectra were obtained in $60 \mathrm{~s} / \mathrm{cell}$, and no damage was observed in the cells following Raman measurement. The spectra had strong bands assigned to $c y t-c$ due to the resonance effect with an excitation wavelength of $532 \mathrm{~nm}$. PCA was successfully applied to analyze spectral differences among the four cancer cells (RERF-LCMS, EBC-1, Lu-65, and RERF-LC-MA) and one normal cell (MRC-5). The loading plot of the PCA model strongly suggests that resonance Raman bands of cyt- $c$ are useful for distinguishing cancer cells from normal cells. The discrimination accuracy of the four cancer cells using PCA analysis was $80 \%$ in the current study. In the discrimination, Raman bands due to cyt- $c$ contributed significantly to $\mathrm{PC} 2$ as well as to the bands due to lipids and/or proteins in PC1. These results suggest an advantage to using a 532-nm excitation wavelength, which gives resonance bands due to cyt-c. Step-wise LDA provided an effective model to classify the histological types of these cells by malignancy type and strain. The discrimination accuracy after cross-validation was $100 \%$. The current results demonstrate the potential of 532-nm-excitation Raman spectroscopy for cell diagnosis.

Raman spectra of cancer cells were obtained without interference of fluorescence in the present study. This result suggests that fluorescence emitted from tissue samples is generated in the extracellular matrix. We are currently developing miniaturized Raman probes with several tens $\mu \mathrm{m}$ of spatial resolution and fluorescent instruments for nonstained tissue imaging. ${ }^{28-31}$ These techniques will make it possible to focus the Raman measurement on the active cellular part of cancer tissues and exclude interference from the extracellular matrix. The current work provides important information and shows the viability of future application of Raman spectroscopy in cancer diagnosis.

\section{Acknowledgments}

This study was supported by Grants-in-Aid for Scientific Research from the Japan Society for the Promotion of Science (JSPS) and from SENTAN (Development of systems and technology for advanced measurement and analysis), Japan Science and Technology Agency (JST).

\section{References}

1. L. G. Collins, C. Haines, R. Perkel, and R. E. Enck, "Lung cancer: Diagnosis and management," Am. Fam. Physician 75(1), 56-63 (2007).

2. W. D. Travis, E. Brambilla, H. K. Müller-Hermelink, and C. C. Harris, "World Health Organization classification of tumours. Pathology and genetics of tumour of the lung, pleura, thymus and heart," Chap. 1 in Tumours of the Lung, pp. 9-50, IARC Press, Lyon, NY (2004).

3. A. Risch and C. Plass, "Lung cancer epigenetics and genetics," Int. J. Cancer 123, 1-7 (2008).

4. L.-P. Choo-Smith, H. G. Edwards, H. P. Endtz, J. M. Kros, F. Heule, H. Barr, J. S. Robinson, Jr., H. A. Bruining, and G. J. Puppels, "Medical applications of Raman spectroscopy: from proof of prin- ciple to clinical implementation," Biopolymers 67, 1-9 (2002).

5. H. B. Hanlon, R. Manoharan, T.-W. Koo, K. E. Shafer, J. T. Motz, M. Fitzmaurice, J. R. Kramer, I. Itzkan, R. R. Dasari, and M. S. Feld, "Prospects for in vivo Raman spectroscopy," Phys. Med. Biol. 45, R1-R59 (2000).

6. Z. Movasaghi, S. Rehman, and I. Rehman, "Raman spectroscopy of biological tissues," Appl. Spectrosc. Rev. 42(5), 493-541 (2007).

7. A. S. Haka, K. E. Shafer-Peltier, M. Fitzmaurice, J. Crowe, R. R. Dasari, and M. S. Feld, "Diagnosing breast cancer by using Raman spectroscopy," Proc. Natl. Acad. Sci. U.S.A. 102(35), 12371-12376 (2005).

8. A. S. Haka, Z. Volynskaya, J. A. Gardecki, J. Nazemi, J. Lyons, D. Hicks, M. Fitzmaurice, R. R. Dasari, J. P. Crowe, and M. S. Feld, "In vivo margin assessment during partial mastectomy breast surgery using Raman spectroscopy," Cancer Res. 66(6), 3317-3322 (2006).

9. Z. Huang, A. McWilliams, H. Lui, D. I. McLean, S. Lam, and H. Zeng, "Near-infrared Raman spectroscopy for optical diagnosis of lung cancer," Int. J. Cancer 107, 1047-1052 (2003).

10. Y. K. Min, T. Yamamoto, E. Kohda, T. Ito, and H. Hamaguchi, "1064 nm near-infrared multichannel Raman spectroscopy of fresh human lung tissues," J. Raman Spectrosc. 36, 73-76 (2005).

11. H. Yamazaki, S. Kaminaka, E. Kohda, M. Mukai, and H. Hamaguchi, "The diagnosis of lung cancer using 1064-nm excited near-infrared multichannel Raman spectroscopy," Radiat. Med. 21(1), 1-6 (2003).

12. N. D. Magee, J. S. Villaumie, E. T. Marple, M. Ennis, J. S. Elborn, and J. J. McGarvey, "Ex vivo diagnosis of lung cancer using a Raman miniprobe," J. Phys. Chem. B 113(23), 8137-8141 (2009).

13. J. W. Chan, D. S. Taylor, S. M. Lane, T. Zwerdling, J. Tuscano, and T. Huser, "Nondestructive identification of individual leukemia cells by laser trapping Raman spectroscopy," Anal. Chem. 80(6), 2180 2187 (2008).

14. A. Y. Lau, L. P. Lee, and J. W. Chan, "An integrated optofluidic platform for Raman-activated cell sorting," Lab Chip 8(7), 11161120 (2008).

15. M. Placzek, B. Przybilla, U. Kerkmann, S. Gaube, and K.-P. Gilbertz, "Effect of ultraviolet (UV) A, UVB or ionizing radiation on the cell cycle of human melanoma cells," Br. J. Dermatol. 156(5), 843-847 (2007).

16. D. I. Pattison and M. J. Davies, In Cancer: Cell Structures, Carcinogens and Genomic Instability, L. P. Bignold, Ed., pp. 131-157, Birkhauser Verlag, Basel, Switzerland (2006).

17. R. J. Swain, G. Jell, and M. M. Stevens, "Non-invasive analysis of cell cycle dynamics in single living cells with Raman microspectroscopy," J. Cell. Biochem. 104(4), 1427-1438 (2008).

18. I. Notingher, I. Bisson, A. E. Bishop, W. L. Randle, J. M. P. Polak, and L. L. Hench, "In situ spectral monitoring of mRNA translation in embryonic stem cells during differentiation in vitro," Anal. Chem. 76(11), 3185-3193 (2004).

19. G. J. Puppels, J. H. F. Olminkhof, G. M. J. Segers-Nolten, C. Otto, F. F. M. de Mul, and J. Greve, "Laser irradiation and Raman spectroscopy of single living cells and chromosomes: Sample degradation occurs with $514.5 \mathrm{~nm}$ but not with $660 \mathrm{~nm}$ laser light," Exp. Cell Res. 195(2), 361-367 (1991).

20. I. Notingher, S. Verrier, H. Romanska, A. E. Bishop, J. M. Polak, and L. L. Hench, "In situ characterization of living cells by Raman spectroscopy," Spectroscopy 16(2), 43-51 (2002).

21. F. Draux, P. Jeannesson, A. Beljebbar, A. Tfayli, N. Fourre, M. Manfait, J. Sulé-Suso, and G. D. Sockalingum, "Raman spectral imaging of single living cancer cells: A preliminary study," Analyst (Cambridge, U.K.) 134(3), 542-548 (2009).

22. I. Notingher, S. Verrier, S. Haque, J. M. Polak, and L. L. Hench, "Spectroscopic study of human lung epithelial cells (A549) in culture: Living cells versus dead cells," Biopolymers 72(4), 230-240 (2003).

23. A. D. Meade, F. M. Lyng, P. Knief, and H. J. Byrne, "Growth substrate induced functional changes elucidated by FTIR and Raman spectroscopy in in-vitro cultured human keratinocytes," Anal. Bioanal. Chem. 387(5), 1717-1728 (2007).

24. R. J. Swain and M. M. Stevens, "Raman microscopy for non-invasive biochemical analysis of single cells," Biochem. Soc. Trans. 35(3), 544-549 (2007).

25. T. G. Spiro and T. C. Strekas, "Resonance Raman spectra of hemoglobin and cytochrome c: Inverse polarization and vibronic scattering," Proc. Natl. Acad. Sci. U.S.A. 69(9), 2622-2626 (1972).

26. K. Hamada, K. Fujita, H. I. Smith, M. Kobayashi, Y. Inouye, and S. 
Oshima et al.: Discrimination analysis of human lung cancer cells associated with histological type and malignancy...

Kawata, "Raman microscopy for dynamic molecular imaging of living cells" J. Biomed. Opt. 13(4), 044027 (2008).

27. D. C. Allison and P. Ridolpho, "Use of a trypan blue assay to measure the deoxyribonucleic acid content and radioactive labeling of viable cells," J. Histochem. Cytochem. 28(7), 700-703 (1980).

28. T. Katagiri, Y. S. Yamamoto, Y. Ozaki, Y. Matsuura, and H. Sato, "High axial resolution Raman probe made of a single hollow optical fiber," Appl. Spectrosc. 63(1), 103-107 (2009).

29. Y. S. Yamamoto, Y. Oshima, H. Shinzawa, T. Katagiri, Y. Matsuura,
Y. Ozaki, and H. Sato, "Subsurface sensing of biomedical tissues using a miniaturized Raman probe: study of thin-layered model samples," Anal. Chim. Acta 619(1), 8-13 (2008).

30. Y. Oshima, C. Furihata, and H. Sato, "Light sheet direct Raman imaging technique for observation of mixing of solvent," Appl. Spectrosc. 63(10), 1115-1120 (2009).

31. Y. Komachi, H. Sato, K. Aizawa, and H. Tashiro, "Micro-optical fiber probe for use in an intravascular Raman endoscope," Appl. Opt. 44(22), 4722-4732 (2005). 